\title{
Drawing Power Law Graphs Using a Local/Global Decomposition ${ }^{1}$
}

\author{
Reid Andersen, ${ }^{2}$ Fan Chung, ${ }^{2}$ and Linyuan $\mathrm{Lu}^{3}$
}

Erratum: The online version of the original article was inadvertantly published with the incorrect article title. The correct title appears above. The publisher regrets the error.

\footnotetext{
${ }^{1}$ A conference version appeared in Proceedings of the Twelfth Annual Symposium on Graph Drawing, 2004. Fan Chung's research was supported in part by NSF Grants DMS 0100472 and ITR 0205061.

2 Department of Mathematics, University of California, San Diego, La Jolla, CA 92093, USA. \{randerse,fan\}@ucsd.edu.

${ }^{3}$ Department of Mathematics, University of South Carolina, Columbia, SC 29208, USA. lu@ maths.sc.edu.

Received December 6, 2004; revised November 5, 2006. Communicated by J. Pach and F. Shahrokhi.

Online publication February 13, 2007.
} 